\title{
Validation of research scales
}

\section{Artola Arita, Vicente; Ramírez, Rudy; Romero, Ángel}

\author{
iD Vicente Artola Arita \\ v.a.artola.arita@umcg.nl \\ Universidad de Groninga, Países Bajos - Antillas \\ Neerlandesas \\ Rudy Ramírez \\ Ministerio de Justicia y Seguridad Pública, Países Bajos \\ - Antillas Neerlandesas \\ Ángel Romero \\ University Research Co., Países Bajos - Antillas \\ Neerlandesas
}

\author{
Alerta \\ Ministerio de Salud, El Salvador \\ ISSN-e: 2617-5274 \\ Periodicidad: Semestral \\ vol. 1, núm. 2, 2018 \\ ralerta@salud.gob.sv \\ Recepción: 19 Marzo 2018 \\ Aprobación: 11 Octubre 2018 \\ Publicación: 12 Diciembre 2018 \\ URL: http://portal.amelica.org/ameli/journal/419/4191909011/ \\ DOI: https://doi.org/10.5377/alerta.v1i2.7140
}

Citación recomendada: Artola Arita V, Ramírez R, Romero Ángel. Validación de escalas para la investigación. Alerta. 2018;1(2):78-80. DOI: $10.5377 /$ alerta.v1i2.7140

Uno de los elementos cruciales para las investigaciones es el instrumento de recolección de datos por ser la concepción operativa a utilizar para recoger los datos y es el paso de transición entre el fundamento teórico y la materialización de ese conocimiento. Estos deben cumplir ciertas características esenciales tales como objetividad, validez, confiabilidad, adecuación entre otras. En este artículo abordaremos la importancia de la validación de escalas adaptadas a El Salvador y una propuesta de pasos para realizarlo.

$\mathrm{Al}$ elegir, adaptar o utilizar una escala es necesario tener en cuenta las siguientes fases propuestas:

\section{a) FASE BASAL}

- Congruencia con el fundamento teórico que se quiere investigar

- Incorporación de todas las variables tomadas en cuenta para estudiar el fenómeno 
- Diseño de la estructura y redacción

b) FASE DE DESARROLLO

- Consistencia interna: Construcción aplicable

- Consistencia externa: Construcción reproducible

c) FASE DE PRUEBA:

- Fiabilidad

- Validación

Se hará una descripción breve de las 3 fases y se propondrá una guía sencilla para la realización de ellas.

La importancia de la Fase Basal, radica en la búsqueda de herramientas o escalas que cumplan los criterios que subsanen las necesidades del investigador ${ }^{1}$ o, al menos, un $80 \%$ de éstas. La fase de desarrollo depende en gran medida de la participación multidisciplinaria que permita la adecuación del contenido de las escalas, pudiendo llevar un tiempo considerable. La fase de prueba es vital para que las escalas sean efectivas, es decir, para que demuestren que lo que se está preguntando es en realidad lo que se está reflejando en las respuestas ${ }^{2}$.

A pesar de que existen escalas validadas para realizar investigaciones, en ocasiones, muchas de ellas están basadas en contextos diferentes a la realidad salvadoreña lo que puede afectar la validez de algunas preguntas por diferentes razones, tales como, compresión de la pregunta, connotación de las palabras, nivel educativo del entrevistado, cultura, traducción, entre muchas otras ${ }^{1,3,4}$; inclusive, en estos casos es necesario realizar pruebas estadísticas para comprobar si la validez es la misma en nuestro contexto ${ }^{5,6,7,8}$. Por otra parte, las investigaciones necesarias en nuestro país pueden diferir de otras realidades, por lo que no siempre se encuentran escalas que se adapten a nuestras necesidades de investigación.

Por lo anteriormente expuesto, es necesario conocer los pasos a seguir para validar escaladas extranjeras y adaptarlas a nuestra realidad -bench marketing- o para crearlas de tal manera que se ajusten a nuestras necesidades de investigación. Los 6 pasos propuestos a seguir para la elección, adaptación o creación ${ }^{9}$ de escalas son los siguientes y pueden intercalarse en las tres fases según necesidad:

1. Búsqueda bibliográfica de escalas que sean compatibles con el objeto a investigar.

2. Seleccionar, Adaptar o Elaborar preguntas que investiguen las variables de interés en nuestro protocolo donde se evalúen todos los indicadores pertinentes por variables dependientes ${ }^{10,11,12}$.

3. Sustentar las preguntas con el marco teórico y los objetivos de la investigación para que las preguntas posean fundamento y puedan ser trianguladas con los resultados y conclusiones ${ }^{10,11,12}$

4. Validar con expertos en el tema para que las preguntas reflejen el conocimiento hasta la fecha desde diferentes puntos de vista. Esto le aportará consistencia y congruencia al instrumento ${ }^{3,13}$.

5. Realizar análisis estadísticos que verifiquen la homogeneidad de las preguntas y la fiabilidad entre ellas. Existen diferentes pruebas para determinar la confiabilidad, tales como, el test Alfa de Cronbach, el índice de homogeneidad a través del índice de correlación de Pearson, el índice de reproductividad $^{14}$ a través del método de Cornell, el coeficiente KR-20, entre otros ${ }^{15}$. La prueba estadística dependerá del tipo de pregunta, escala y respuesta (dicotómica, en escala, lineal, entre otros) $)^{5,6,7,8,16}$.

6. Realizar una prueba piloto enmarcada como parte del protocolo de investigación ${ }^{2}$.

$\mathrm{Al}$ tomar en cuenta estas fases y la guía de pasos dinámicos propuestos, podremos construir escalas que se adecúen a nuestra realidad y a nuestras necesidades de investigación para obtener resultados más fidedignos 


\section{ACLARACIÓN}

Los 3 autores contribuyeron de forma equitativa en la construcción del documento.

\section{REFERENCIAS BIBLIOGRÁFICAS}

1. Hinkin T. A Review of Scale Development Practices in the Study of Organizations. Journal of Management. 1995 Octubre; 21(5).

2. Fowler F. Improving Survey Questions: Design and Evaluation. 1st ed. Publications S, editor. Thousand Oaks: Thousand Oaks; 1995

3. Morgado F, Meireles J, Neves C, Amaral A, Ferreira M. Scale development: ten main limitations and recommendations to improve future research practices. Psicologia: Reflexão e Crítica. 2017 Enero; 30(3).

4. McPhail S. Alternative Validation Strategies: Developing New and Leveraging Existing Validity Evidence. 1st ed. San Francisco: John Wiley \& Sons, Inc; 2007.

5. Lawshe C. A quantitative approach to content validity. Personnel Psychology. 1975 Diciembre; 28(4).

6. Lynn M. Determination and quantification of content validity. Nursing Research. 1986 Noviembre; 35(6).

7. Cohen J. A Coefficient of Agreement for Nominal Scales. Educational and Psychological Measurement. 1960 Abril; 20(1).

8. Wynd C, Schmidt B, Schaefer M. Two quantitative approaches for estimating content validity. Western Journal of Nursing Research. 2003 Agosto; 25(5).

9. Sanchez R, Echeverry J. Validación de Escalas de Medición en Salud. Revista de Salud Pública. 2004 Noviembre; 6(3).

10. Haynes S, Richard D, Kubany E. Content validity in psychological assessment: A functional approach to concepts and methods. Psychological Assessment. 1995 Septiembre; 7(3).

11. Raykov T, Marcoulides G. Introduction to Psychometric Theory. 1st ed. Nueva York: Taylor \& Francis Group; 2011.

12. Kline P. Handbook of Psychological Testing. 2nd ed. Londres: Routledge; 1999.

13. DeVellis R. Scale Development: Theory and Application. 1st ed. Los Angeles: Sage Publications; 2012.

14. Bland J, Altman D. Validating scales and indexes. British Medical Journal. 2002 Marzo; 324.

15. Sampieri R, Collado C, Lucio P. Metodología de la Investigación. 4th ed. Alayón RDB, editor. México: Mc GrawHill; 2006.

16. Franklin E. Escalas de Medición. In Guerrero P, editor. Auditoría Administrativa. México: Person Educación de México; 2007. p. 493-507. 